\title{
Incorporation of Finite Element Analysis into Annual Energy Loss Estimation for Permanent Magnet Wind Turbine Generators
}

\author{
Henriksen, Matthew Lee; Jensen, Bogi Bech
}

Published in:

Proceedings of the 2013 IEEE International Electric Machines \& Drives Conference

Link to article, DOI:

10.1109/IEMDC.2013.6556150

Publication date:

2013

Link back to DTU Orbit

Citation (APA):

Henriksen, M. L., \& Jensen, B. B. (2013). Incorporation of Finite Element Analysis into Annual Energy Loss Estimation for Permanent Magnet Wind Turbine Generators. In Proceedings of the 2013 IEEE International Electric Machines \& Drives Conference (pp. 557 - 563). IEEE. https://doi.org/10.1109/IEMDC.2013.6556150

\section{General rights}

Copyright and moral rights for the publications made accessible in the public portal are retained by the authors and/or other copyright owners and it is a condition of accessing publications that users recognise and abide by the legal requirements associated with these rights.

- Users may download and print one copy of any publication from the public portal for the purpose of private study or research.

- You may not further distribute the material or use it for any profit-making activity or commercial gain

- You may freely distribute the URL identifying the publication in the public portal 


\title{
Incorporation of Finite Element Analysis into Annual Energy Loss Estimation for Permanent Magnet Wind Turbine Generators
}

\author{
Matthew L. Henriksen and Bogi B. Jensen \\ Center for Electric Power and Engineering \\ Technical University of Denmark \\ 2800 Kongens Lyngby, Denmark \\ Email: mlee@elektro.dtu.dk, bbj@elektro.dtu.dk
}

\begin{abstract}
Several methods of estimating the annual energy losses for wind turbine generators are investigated in this paper. Utilizing a high amount of transient simulations with motion is first demonstrated. Usage of a space-time transformation for prediction of iron losses is also explored. The methods, which use varying amounts of simulation results and computational resources, are compared based on time required and consistency in varying wind climates.
\end{abstract}

\section{INTRODUCTION}

The international level of installed wind power capacity continues to grow [1]. As the power levels of wind turbines rise, and the installation of wind turbines offshore becomes increasingly popular, the academic and industrial communities are being faced with a myriad of opportunities, challenges, and problems.

Amongst such challenges is the choice of an optimal wind turbine drivetrain, which is a frequently debated topic. Manufacturers today are certainly not at a concensus on the issue, as can be ascertained by viewing the availability of squirrel cage and doubly-fed induction machines, as well as permanent magnet and electrically-excited synchronous machines on the market [2], [3], [4], [5], [6]. Adding the choices between direct-drive and various levels of gearing for the drivetrains, and the use for a flexible, fast, and comprehensive design comparison process becomes apparent.

While this is not an entirely new idea, it is very interesting to discuss and propose improvements for such design comparison processes. Along these lines, the current paper contributes to the discussion by demonstrating a search for a fast and dependable method of estimating the annual energy losses $(A E L)$ due to iron losses for a permanent magnet wind turbine generator. Finite element analysis (FEA) for estimating the losses is considered, and compared to other examples in literature it is expected that this approach will give a better approximation. The balance between speed and relative accuracy is explored by comparing several different evaluation methods.

After expanding on the motivation of the current paper, as well as a brief consideration of earlier work, the principles of variable-speed wind turbine operation are discussed. This is important for the current work because it defines the simulation settings which are used while estimating the iron losses. Details regarding the example wind turbine generator are provided, followed by iron loss estimation results from the employed commercial FEA package's built-in solver, as well as results obtained using a handful of static $2 \mathrm{D}$ results along with a space-time transformation (STT). These results are then utilized in various ways to estimate the $A E L$ due to iron losses, and the various methods are then discussed and compared.

\section{A. Motivation}

The goal of a wind turbine is to make money for those who have invested in its construction and operation. With that in mind, the anticipated cost of energy $(C O E)$ is an important metric in terms of comparing potential drivetrain solutions at the early design stages. All costs related to purchase and construction of the wind turbine form the $C A P E X$, while all costs associated with maintenance and operation constitue the $O P E X$ for the wind turbine. The sum of these two annualized over the wind turbine lifetime, divided by the wind turbine's annual energy production $(A E P)$, gives the $C O E$.

$$
C O E=\frac{C A P E X+O P E X}{A E P}
$$

There are a huge number of factors that enter these estimations. In terms of choosing a drivetrain configuration, the cost of the components and the annual losses will definitely enter the equation.

Losses occur to some degree in all wind turbine drivetrain components. The relationship between the losses and the $A E P$ is simply that the annual energy gathered by the blades, minus the annual losses, gives the $A E P$. Looking to the generator, the primary sources of losses are ohmic losses and iron losses. Estimation of the $A E P$ therefore requires that the annual energy losses due to iron can be estimated first. It is of course important to estimate the annual energy losses due to all loss mechanisms, but the paper at hand is solely focused on how to handle the problem of the iron losses.

In this work, the simulation time required to achieve a prediction of the $A E L$ based on FEA is investigated. It is anticipated that one or more of the demonstrated methods can be used for various design comparison studies, based on the level of accuracy and the required robustness of the 
method. For this reason, the $A E L$ due to iron losses is predicted for several wind regimes, representing some typical locations for wind farm construction. The possibility of performing a reduced number of simulations and interpolating or extrapolating for the remainder of the iron loss data exists, and this is demonstrated as one way to reduce the required computational time. In the interest of producing even faster results, abandonment of transient simulations in favor of static simulations in conjunction with a space-time transformation is considered as well.

\section{B. Prior Work}

Wind turbine generator design studies can be found in high numbers throughout the literature. Several examples can be noted, in which the losses at partial load have been considered and used in producing an estimate of, or at least similar to, the cost of energy [7]. Commonly, the iron loss information from a manufacturer of laminations is used, while multiplying by an enhancement factor to account for the variation between the loss measurement conditions and the expected application conditions [8], [9], [10], [11]. The effect of the variation of frequency is thereby captured, but the local variation in flux density due to armature currents is disregarded, as is the effect of the flux density vector rotating in some regions of the iron rather than just pulsating.

For the case of permanent magnet synchronous machines (PMSM), the iron losses are particularly difficult to predict under variable speed and variable load operation. Finite element analysis (FEA) has historically proven to be a useful tool for this [12]. Nevertheless, the prospect of performing the required simulations under varying operational conditions may deter many designers.

\section{VARIABle SPEed Wind TURbine Operation}

It is important to present some fundamental aspects of variable speed wind turbine operation, in order for the simulated conditions and subsequent conclusions to be understood. Thus, a basic control strategy for a variable speed wind turbine is presented in this section, followed by an explanation of the usage of Weibull parameters to describe different wind climates.

\section{A. Control of Variable Speed Wind Turbines}

Variable speed operation of a wind turbine gives the advantage that the blade speed can be adjusted in order to maximize the power gathered by the blades. This, along with optimal pitching of the blades, manifests itself in the achievement of the highest possible coefficient of power $C_{p}$. As shown in equation 2, the coefficient of power appears in direct proportion to the total gathered power,

$$
P_{\text {blades }}=\frac{1}{2} C_{p}(\lambda) A \rho_{\text {air }} v^{3}
$$

where $A$ is the swept area of the blades, $\rho_{\text {air }}$ is the density of air at standard temperature and pressure, and $v$ is the wind speed. The coefficient of power depends, as indicated in equation 2 , on the tip speed ratio $\lambda$. The tip speed ratio is given by equation 3 ,

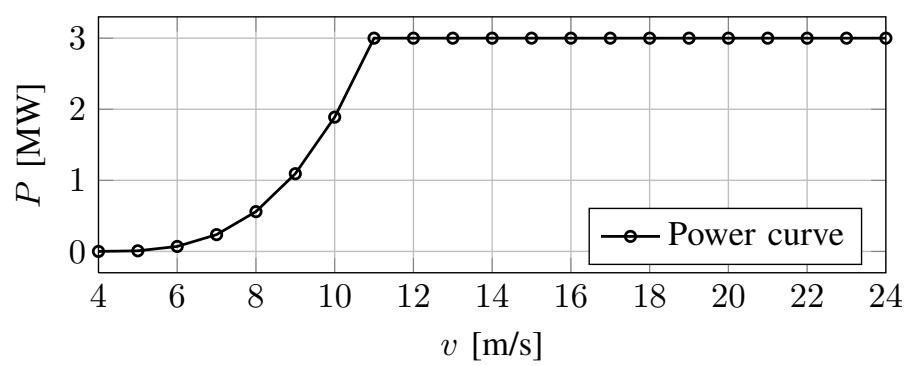

(a)

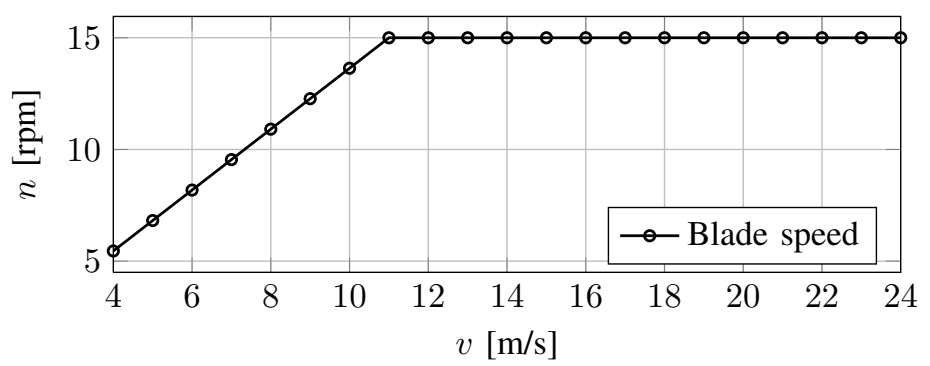

(b)

Fig. 1. Power output (a) and blade speed (b) as functions of wind speed

$$
\lambda=\frac{\omega R}{v}
$$

which takes the product of the blade angular frequency $\omega$ and the blade radius $R$ divided by the wind speed. As dictated by equation 3 , to maintain the optimal coefficient of power while in variable speed mode, the rotational speed of the blades thus takes on a linear relationship with the wind speed.

For the purpose of control, the wind regime can be divided into variable speed and fixed speed regimes [13]. The point of division between these two wind regimes is the so-called rated wind speed. After the wind reaches this level, the blades begin to be pitched such that excess power is spilled by the blades. This maintains a constant power output when the wind blows between the rated and cut-out wind speeds.

For a permanent magnet machine, the voltage varies in direct proportion to the rotational speed. Equation 2 indicates that the incoming power is proportional to the wind speed cubed. It follows then that the current must follow the wind speed squared. Under the assumption that the no-load condition for the generator exists at the cut-in wind speed, table I provides the operating conditions for variable speed mode, as dictated by equations 4 through 6 .

$$
\begin{aligned}
f(v) & =f_{\text {rated }} \frac{v}{v_{\text {rated }}} \\
V(v) & =V_{\text {rated }} \frac{v}{v_{\text {rated }}} \\
I(v) & =I_{\text {rated }}\left(1-\left(\frac{v-v_{\text {rated }}}{v_{\text {rated }}-v_{\text {cutin }}}\right)^{2}\right)
\end{aligned}
$$


TABLE I. VARIABLE SPEED OPERATION

\begin{tabular}{llll}
\hline$v[\mathrm{~m} / \mathrm{s}]$ & $f[\mathrm{~Hz}]$ & $V_{r m s}[\mathrm{~V}]$ & $I_{r m s}[\mathrm{~A}]$ \\
\hline 4 & 43.6 & 250.9 & 0.0 \\
5 & 54.5 & 313.6 & 666.0 \\
6 & 65.5 & 376.4 & 1229.5 \\
7 & 76.4 & 439.1 & 1690.6 \\
8 & 87.3 & 501.8 & 2049.2 \\
9 & 98.2 & 564.5 & 2305.3 \\
10 & 109.1 & 627.3 & 2459.0 \\
11 & 120.0 & 690.0 & 2510.2 \\
\hline
\end{tabular}

TABLE II. THE NINE WIND CLIMATES SELECTED FOR THE STUDY, REPRESENTED IN TERMS OF THEIR WEIBULL PARAMETERS

\begin{tabular}{lll}
\hline Site description & Shape factor $k$ & Scale factor $A$ \\
\hline Low k, low A & 0.5 & 4.0 \\
Low k, medium A & 0.5 & 8.0 \\
Low k, high A & 0.5 & 12.0 \\
Medium k, low A & 1.5 & 4.0 \\
Medium k, medium A & 1.5 & 8.0 \\
Medium k, high A & 1.5 & 12.0 \\
High k, low A & 2.5 & 4.0 \\
High k, medium A & 2.5 & 8.9 \\
High k, high A & 2.5 & 12.0 \\
\hline
\end{tabular}

\section{B. Wind Climates}

Wind climates are commonly described in terms of a Weibull distribution, where the shape factor $k$ and scale factor $A$ are the two defining parameters. From the Weibull distribution, the probability that the wind speed will lie within the interval given by wind speeds $v_{i}$ and $v_{i+1}$ can be found by equation 7 .

$$
p=e^{\left[-\left(\frac{V_{i}}{A}\right)^{k}\right]}-e^{\left[-\left(\frac{V_{i+1}}{A}\right)^{k}\right]}
$$

Nine representative sets of Weibull parameters have been selected based on observation of the European Wind Atlas, in order to demonstrate the loss estimation for different wind climates [14]. The European Wind Atlas is based on wind measurements at many locations, and uses the Weibull parameters to describe the observed wind climates. To summarize, typical values of $k$ are 0.5 to 2.5 , while for $A$ the values tend to fall between 4 and 15. With an intent to cover the possible combinations of high and low $k$ and $A$ values, the wind climates described in table II are used in this study. Figure 2 gives the probability of the wind speed falling within 25 different intervals, starting with $0-1 \mathrm{~m} / \mathrm{s}$ and finishing with $24-25 \mathrm{~m} / \mathrm{s}$.

Viewing figure 2 can lead to a general understanding of the influence of the shape factor $k$ and scale factor $A$. The scale factor $A$ is related to the mean value of the wind speed, so as it increases the probabilities of higher wind speeds also increase. The value of $k$, on the other hand, influences the likelihood of the wind speeds to fall near the value of $A$. Thus, for high values of $k$ the wind distribution tends to concentrate about the value of the scale factor. For low values of $k$ the wind distribution becomes spread more evenly across all wind speeds.

\section{The Example Wind Turbine Generator}

For this study, the example of a 12-pole generator, dimensioned for operation under the conditions described in table

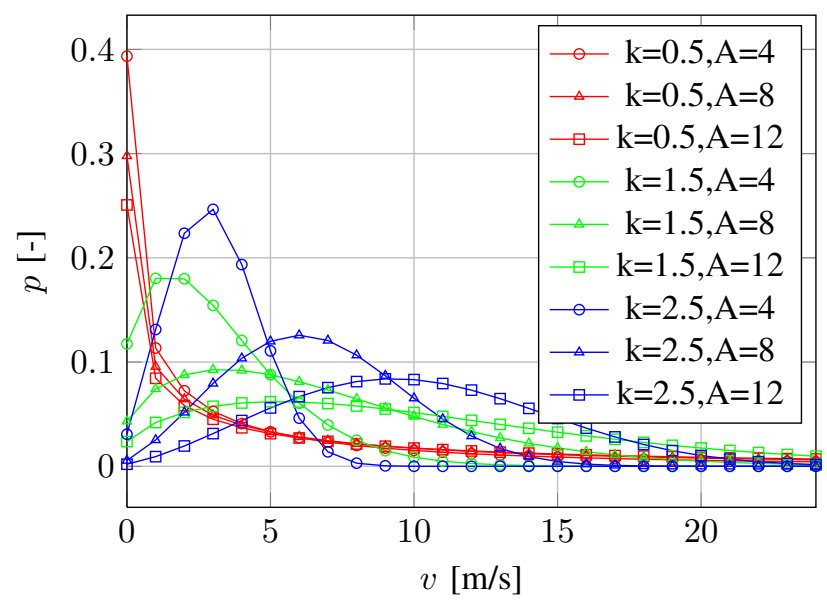

Fig. 2. Weibull probability density functions for the nine example wind climates considered in this study

TABLE III. OPERATIONAL CONDITIONS FOR THE EXAMPLE WIND TURBINE

\begin{tabular}{lll}
\hline Paremeter & Description & Value \\
\hline$v_{\text {cutin }}$ & Cut in wind speed & $4 \mathrm{~m} / \mathrm{s}$ \\
$v_{\text {rated }}$ & Rated wind speed & $11 \mathrm{~m} / \mathrm{s}$ \\
$v_{\text {cutout }}$ & Cut-out wind speed & $25 \mathrm{~m} / \mathrm{s}$ \\
\hline
\end{tabular}

IV, is considered. Figure 3 gives a view of the cross section, as well as a close up of one pole. It will later be explained that due to the winding arrangement three 'types' of teeth can be found, which are also indicated in figure 3 (b).

The generator is assumed to be driven through a gearbox by appropriately sized blades. The specifics of these are not relevant for this study, but table III does give the important details regarding cut-in, rated, and cut-out wind speeds. These must be considered along with the losses and the wind climate while predicting the AEL.

\section{Iron Loss Estimation Throughout Power CURVE}

To assess the effect of varying the armature current and the generator's rotational speed on the iron losses, several points along the wind turbine power curve are assessed. The details regarding the laminations are provided in table V. It has been shown in literature that compared to simulated results, the

TABLE IV. GENERATOR DIMENSIONS

\begin{tabular}{llll}
\hline Parameter & Symbol & Value & Units \\
\hline Rated Power & $P_{\text {rated }}$ & 3 & {$[\mathrm{MW}]$} \\
Rated speed & $n_{\text {rated }}$ & 1200 & {$[\mathrm{rpm}]$} \\
Rated voltage & $V_{\text {rated }}$ & 690 & {$[\mathrm{~V}]$} \\
Air gap diameter & $D_{s}$ & 0.74 & {$[\mathrm{~m}]$} \\
Stack length & $l_{s}$ & 0.8 & {$[\mathrm{~m}]$} \\
Air gap length & $l_{g}$ & 0.004 & {$[\mathrm{~m}]$} \\
Pole pairs & $p$ & 6 & {$[-]$} \\
Slots/pole/phase & $q$ & 3 & {$[-]$} \\
Magnet height & $h_{P M}$ & 0.012 & {$[\mathrm{~m}]$} \\
Magnet pitch fraction & $a_{P M}$ & 0.8 & {$[-]$} \\
Stator coreback thickness & $d_{c b s}$ & 5.00 & {$[\mathrm{~cm}]$} \\
Rotor coreback thickness & $d_{c b r}$ & 5.00 & {$[\mathrm{~cm}]$} \\
Tooth width & $w_{s s}$ & 1.12 & {$[\mathrm{~cm}]$} \\
Slot width & $w_{s t}$ & 1.00 & {$[\mathrm{~cm}]$} \\
Slot depth & $d_{s s}$ & 6.60 & {$[\mathrm{~cm}]$} \\
\hline
\end{tabular}




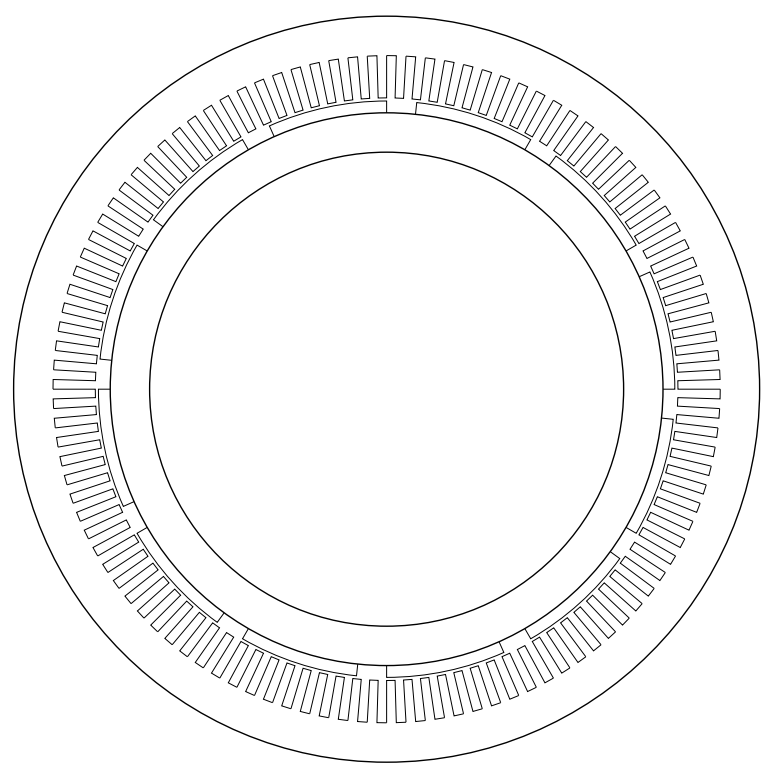

(a)

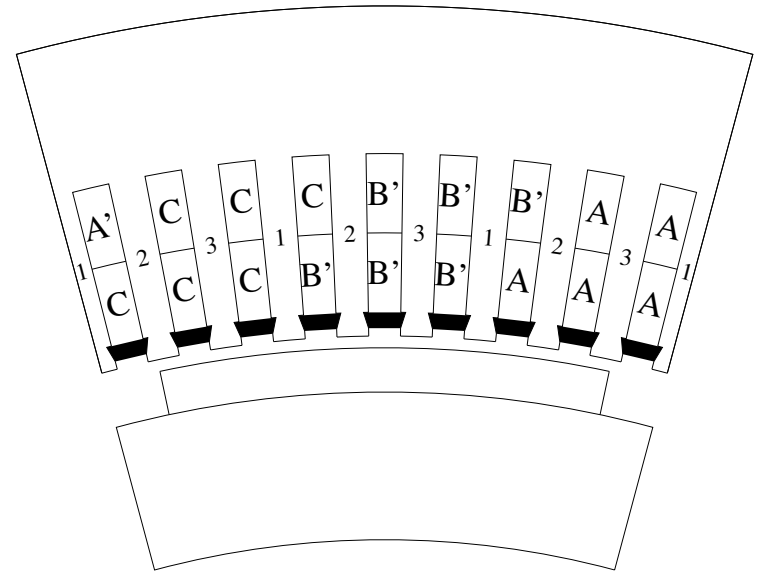

(b)

Fig. 3. Pictures of the generator (a) machine cross section (b) close up view of one pole, with labeled coils and teeth types for the space-time transformation

TABLE V. INFORMATION ABOUT THE LAMINATIONS

\begin{tabular}{lll}
\hline Parameter & Description & Value \\
\hline Name & M400-50A & NA \\
$k_{h}$ & Hysteresis factor & $1.098 \mathrm{E}-2$ \\
$k_{e}$ & Eddy factor & $7.921 \mathrm{E}-5$ \\
$\alpha$ & Hysteresis frequency exponent & 1.234 \\
$\beta$ & Hysteresis flux density exponent & 1.775 \\
$k_{d f}$ & Design factor & 1.7 \\
\hline
\end{tabular}

manufacturing process for large generators tends to result in significantly increased iron losses for a constructed machine [15]. A design factor $k_{d f}$ of 1.7 is therefore adopted. All simulated iron loss results have been multiplied by the design factor in order to obtain the presented values.

\section{A. Transient with Motion Analysis via MagNet}

If available, one obvious measure is to employ a commercial finite element analysis package with built-in iron loss evaluation. In this study Infolytica MagNet, which has been verified as having the potential to accurately estimate iron
TABLE VI. IRON LOSSES OBTAINED BY THE TWO EMPLOYED METHODS

\begin{tabular}{|c|c|c|c|c|c|c|}
\hline \multirow{3}{*}{ Wind Speed $[\mathrm{m} / \mathrm{s}]$} & \multicolumn{6}{|c|}{ Estimated iron losses $[\mathrm{kW}]$} \\
\hline & \multicolumn{3}{|c|}{ Time-stepping FEA } & \multicolumn{3}{|c|}{ STT } \\
\hline & Hys & Eddy & Total & Hys & Eddy & Total \\
\hline 4 & 5.19 & 1.11 & 6.30 & 6.47 & 1.14 & 7.61 \\
\hline 5 & 7.21 & 1.79 & 9.00 & 8.74 & 1.80 & 10.54 \\
\hline 6 & 9.60 & 2.66 & 12.26 & 11.37 & 2.66 & 14.03 \\
\hline 7 & 12.33 & 3.73 & 16.06 & 14.29 & 3.71 & 18.00 \\
\hline 8 & 15.26 & 5.00 & 20.26 & 17.42 & 4.94 & 22.36 \\
\hline 9 & 18.27 & 6.43 & 24.71 & 20.65 & 6.33 & 26.98 \\
\hline 10 & 21.24 & 8.02 & 29.27 & 23.89 & 7.88 & 31.76 \\
\hline 11 & 24.06 & 9.74 & 33.80 & 27.01 & 9.56 & 36.57 \\
\hline
\end{tabular}

losses, has been used for this purpose [16]. The actual iron loss calculation method is not explicitly available to users due to some proprietary aspects [17]. It is however based on the Steinmetz equation in the following form, where the specific iron loss is expressed as:

$$
p_{f e}=k_{h} f^{\alpha} B^{\beta}+k_{e}(f B)^{2}
$$

The first term in equation 8 represents losses due to hysteresis, as well as anomalous losses. The second term represents the losses due to eddy currents [17]. The coefficients in equation 8 are reported as obtained through curve fitting of loss measurements at $50 \mathrm{~Hz}, 100 \mathrm{~Hz}, 200 \mathrm{~Hz}, 400 \mathrm{~Hz}, 1000 \mathrm{~Hz}$ and $2500 \mathrm{~Hz}$, with an $\mathrm{R}^{2}$ of 0.9986 .

The results of simulating operation at integral wind speeds from $4-11 \mathrm{~m} / \mathrm{s}$ can be seen in table VI. Because this evaluation is performed on a per-mesh basis, includes a large number of samples, and explicitly analyzes all desired operating points, it is from this point forward taken as the standard to which other, lighter methods should be compared. The downside, investigated later in the paper, is the large amount of time required to perform these eight simulations.

\section{B. Space-Time Transformation}

Usage of a space-time transformation has been demonstrated to be effective in generating iron loss data in good agreement with time stepping FEA solutions for PM machines [18]. This method utilizes a low number of static simulations, combined with symmetry and periodicity in the machine, in order to synthesize time-varying waveforms for flux density in various locations throughout the machine. Interested readers are referred to several examples in literature to learn more [19], [20], [18].

As opposed to the aforementioned commercial method which considers the losses on a per-mesh basis, this method will be employed with a course discretization of the model. For execution of this method on the example machine for this paper, the stator, shown for one pole in figure 3 (b), is subdivided into several regions:

- Tooth tip

- Tooth body

- Inner coreback over slot

- Outer coreback over slot 
- Inner coreback over tooth

- Outer coreback over tooth

Taking the winding arrangement into account, three types of teeth can be observed based on the coil sides in the slots on either side of the tooth. Because the machine is synchronous, and because there are three of each tooth type in one pole for this machine, a single static solution of one pole gives three time-delayed values for the flux density of each tooth type. By repeating the simulation five times over the time required for the rotor to move across three slots, 15 equally-spaced points along half of the flux density waveform for each tooth type are obtained. Odd periodicity gives 15 more points, for a total of 30 points over the whole flux density waveform.

Figure 4 helps with visualizing the employed method. In the first static simulation the values associated with the three circle markers in figure 4 were obtained, and their spatial displacement was transformed into the time domain. Repeating this process five times, and using the odd periodic boundary condition, the dashed, red plot was obtained. The solid, black plot indicates the radial flux density in tooth type 1 , as obtained during a transient with motion simulation. Clearly the waveform obtained via the static simulations is a good match with the one obtained with the transient simulation. Similar results are obtained in the same manner for the other two types of teeth, and as well in the stator coreback.

The next step is finding the frequency components of the obtained flux density waveforms. If the series of simulations is correctly configured, the points along the flux density waveform are evenly spaced. In this case it is possible to employ a fast fourier transform on the wave, thus providing coefficients for the first $n / 2-1$ harmonic orders, where $n$ is the number of points on the wave being processed. For the case in question the value of $n$ is 30 , and thus harmonics up to the 14th will be included. Equation 9 is then employed to find the iron loss contributions from the present harmonics.

$$
p_{f e, v}=k_{h} f_{v}^{\alpha} B_{v}^{\beta}+k_{e}\left(f_{v} B_{v}\right)^{2}
$$

Equation 9 is used independently on the radial and tangential components of the flux density. This has been suggested as a way to help with incorporation of the effect of a rotational magnetic flux density vector in the iron [21]. A source of error and potential discrepancy between prediction of iron losses with equations 8 and 9 is the treatment of term 1 . In equation 9 harmonic decomposition is used on this term, while MagNet handles this term differently [17]. Usage of harmonic decomposition is not technically correct for non-linear phenmonon such as the hysteresis and anomalous losses, but it is an inaccuracy which can be tolerated if the fundamental component is the dominate term [22].

Table VI provides the losses for all operating points of interest obtained via this method. It is possible to see that the agreement with Infolytica's solution is strong, but does suffer somewhat for the lightly loaded, low speed operational points. This is important to note, moving forward to the following section in which the annual energy losses for various wind climates are investigated.

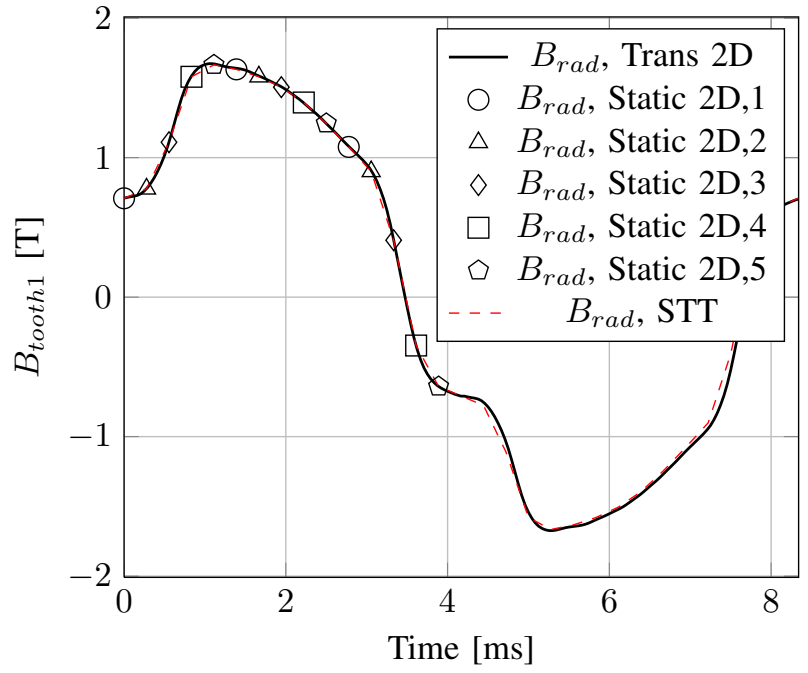

Fig. 4. The radial flux density in tooth type 1, as observed from a transient simulation, and as reconstructed from 5 static simulations and usage of the time-space transformation

TABLE VII. DESCRIPTION OF METHODS AND TIME REQUIRED

\begin{tabular}{llll}
\hline Method & Simulations & FEA time & Postprocessing time \\
\hline 1 & 8 trans 2D+motion & $122 \mathrm{~s}$ & {$[-]$} \\
2 & 1 trans 2D+motion & $20 \mathrm{~s}$ & {$[-]$} \\
3 & 1 trans 2D+motion & $13 \mathrm{~s}$ & {$[-]$} \\
4 & 2 trans 2D+motion & $34 \mathrm{~s}$ & {$[-]$} \\
5 & 5 static simulations & $16 \mathrm{~s}$ & $2 \mathrm{~s}$ \\
6 & 10 static simulations & $21 \mathrm{~s}$ & $5 \mathrm{~s}$ \\
\hline
\end{tabular}

\section{Estimation of ANNUAL ENERGy LOSSES}

Having estimated the iron losses $P_{f e}$ as shown in table VI, and having found the probabilities $p$ of the wind speed falling within intervals 1 through $\mathrm{n}$, the annual energy losses due to iron losses can be found according to equation 10 :

$$
A E L=\sum_{i=1}^{n} 8760 * p_{i} * \frac{1}{2}\left(P_{f e, i}+P_{f e, i+1}\right)
$$

This section of the paper compares six methods for producing a result for equation 10. Table VII describes the six methods, and also gives the required time experienced by the authors. Since execution times are being compared, it is relevant to note some details regarding the employed PC workstation, which can be found in table VIII. Table IX provides a summary of results obtained with all six methods, and the relative error when compared to Method 1.

\section{A. Method 1 - Using all Transient with Motion Solutions}

Method 1 is the most time consuming, detailed method for estimating the iron losses. The results of eight transient with motion simulations are required. This series of results was

TABLE VIII. WORKSTATION DETAILS

\begin{tabular}{ll}
\hline Processor & Intel(R) Core(TM) i5 CPU M 560 2.67GHz \\
Memory (RAM) & 4.00 GB \\
Operating system & 64-bit Windows 7 Enterprise \\
\hline
\end{tabular}


TABLE IX. ESTIMATED ANNUAL ENERGY LOSSES DUE TO IRON

\begin{tabular}{|c|c|c|c|c|c|c|c|c|c|c|c|c|c|}
\hline \multirow[b]{2}{*}{$\mathrm{k}$} & \multirow[b]{2}{*}{ A } & \multicolumn{2}{|c|}{ Method 1} & \multicolumn{2}{|c|}{ Method 2} & \multicolumn{2}{|c|}{ Method 3} & \multicolumn{2}{|c|}{ Method 4} & \multicolumn{2}{|c|}{ Method 5} & \multicolumn{2}{|c|}{ Method 6} \\
\hline & & AEL [MWh] & $\Delta[\%]$ & AEL [MWh] & $\Delta[\%]$ & AEL [MWh] & $\Delta[\%]$ & AEL [MWh] & $\Delta[\%]$ & AEL [MWh] & $\Delta[\%]$ & AEL [MWh] & $\Delta[\%]$ \\
\hline 0.5 & 4 & 54.40 & {$[-]$} & 44.30 & -18.57 & 56.83 & 4.47 & 53.84 & -1.03 & 61.72 & 13.45 & 59.40 & 9.19 \\
\hline 0.5 & 8 & 64.84 & {$[-]$} & 52.49 & -19.05 & 67.30 & 3.80 & 64.23 & -0.93 & 73.05 & 12.67 & 70.66 & 8.98 \\
\hline 0.5 & 12 & 66.98 & {$[-]$} & 54.09 & -19.24 & 69.34 & 3.53 & 66.38 & -0.89 & 75.25 & 12.35 & 72.94 & 8.90 \\
\hline 1.5 & 4 & 38.67 & {$[-]$} & 34.29 & -11.33 & 44.29 & 14.54 & 37.96 & -1.84 & 48.41 & 25.19 & 43.54 & 12.60 \\
\hline 1.5 & 8 & 124.73 & {$[-]$} & 102.34 & -17.95 & 131.40 & 5.35 & 122.97 & -1.41 & 142.81 & 14.49 & 136.23 & 9.22 \\
\hline 1.5 & 12 & 167.20 & {$[-]$} & 134.42 & -19.60 & 172.27 & 3.03 & 165.59 & -0.96 & 186.89 & 11.78 & 181.66 & 8.65 \\
\hline 2.5 & 4 & 26.96 & {$[-]$} & 25.65 & -4.87 & 33.28 & 23.44 & 26.71 & -0.92 & 36.52 & 35.45 & 31.56 & 17.05 \\
\hline 2.5 & 8 & 132.41 & {$[-]$} & 110.37 & -16.64 & 141.96 & 7.21 & 129.66 & -2.08 & 154.52 & 16.70 & 144.90 & 9.43 \\
\hline 2.5 & 12 & 210.41 & {$[-]$} & 168.46 & -19.94 & 215.83 & 2.58 & 208.14 & -1.08 & 234.09 & 11.25 & 228.02 & 8.37 \\
\hline
\end{tabular}

obtained in 2 minutes and 2 seconds. For many cases this may be an acceptable amount of time. This is, however, quite a long time for the early design stages of a machine being designed to achieve a low cost of energy. In that case the result of equation 10 is of direct interest, and a designer would like to know how every slight alteration would affect this value. Especially looking to the case where optimal design is employed and the annual energy losses are required for formation of an objective function, this evaluation could become a significant bottleneck.

\section{B. Methods 2 and 3 - Using one Transient with Motion Solution}

In Methods 2 and 3, solely the transient with motion simulation results at no-load and full-load, respectively, were used. The iron losses at the remaining wind speeds were extrapolated, where only the frequency terms in equation 8 were adjusted. This procedure cuts down the required simulation time drastically, but the effect of the armature current is neglected for all operational points except for at the point of simulation being used. Table IX indicates, interestingly, that strong agreement can be found between Methods 1 and $2+3$ for certain types of wind regimes.

Looking closer, the agreement improves when the wind regime dictates that signficant time would be spent near the wind speed from which the single simulation result is taken. Thus, Method 2 is useful at sites where the wind is concentrated at low levels. Method 3, on the other hand, takes the full load result $(v=11 \mathrm{~m} / \mathrm{s})$. Accordingly, the agreement with Method 1 is strong for sites characterized by high winds. In this case the effect of the full load armature reaction on the flux density in the iron is incorporated. Although this effect will manifest itself differently at partial load, it gives a better, more universally applicable sample than extrapolating based on the simulation at no-load. The largest errors between Methods 3 and 1 appear as the wind speeds become more concentrated at low values.

\section{Method 4 - Using Two Transient with Motion Solutions}

As a compromise between speed and flexibility, Method 4 is investigated. In this method the results from no-load and full-load are taken. A third point is obtained for zero iron losses at a wind speed of zero, and a cubic fit of the three points was used to find the iron losses at the remaining wind speeds.

Viewing table IX, it can be seen that Method 4 generally gives great agreement with Method 1. While in terms of time, this represents a significant improvement over the initial case where eight simulations were employed, this method still would require around 30 seconds to evaluate a single candidate design at the workstation used for this study. As concerns the certainty of the results, two transient with motion simulations appear to be sufficient to obtain the same AEL result within $2 \%$ as Method 1, regardless of the details of the wind climate.

\section{Method 5 - Using 5 Static Simulations}

While Method 1 serves as a benchmark, representing for this study the most accurate and detailed AEL prediction method, Methods 3 and 4 demonstrated that simulation of one or two operation points will typically give very similar results in a much shorter time. Taking this idea to the next level, Method 5 utilizes the STT at full load and extrapolates the results to the partial load operational points. The method requires just five static $2 \mathrm{D}$ simulations.

Table IX shows that Method 5 is capable of matching Method 1 within $12 \%$ for sites where the wind has a tendency to be concentrated at high values. For some cases this may be sufficient, but it must always be noted that this result is highly dependent on the wind regime, and if care is not taken the AEL prediction could exhibit a huge difference (see $k=2.5$, $A=4$ ) from what would be optained via detailed simulation of all operational points of interest.

\section{E. Method 6 - Using 10 Static Simulations}

As a final measure, Method 4 is adapted for usage with results from the STT. In Method 6, the generator's operation is analyzed with the STT at no load and full load, requiring a total of 10 static 2D simulations. After postprocessing, the total time required is still low in comparison with Methods 1-4, which are based on the usage of transient with motion solutions.

The inclusion of a second operating point has a strong effect on the robustness of the calculation method, in comparison with Method 5. Now, the AEL results could be trusted to within $10 \%$ for a much wider range of wind climates. Relatively poor agreement can still be observed for sites with low $A$ and high $k$, so the user must still take care in deciding whether or not this prediction method is suitable. When comparing Methods 6 and 4, Method 4 gives much better agreement with Method 1 , while the advantage of Method 6 in speed is only around $25 \%$.

\section{F. Comments}

As an overall result, this comparison shows that detailed simulation of a large number of operating points along the 
power curve should not be required, at least for the purpose of estimating the annual iron losses. Regarding the time required for the methods based on the STT, it should be mentioned that for other winding layouts the comparison could turn out differently. Furthermore, reduction of the postprocessing time has not been a focus in this work, and that is of course one area where there could be potential to save time.

\section{CONCLUSION}

This paper has investigated several methods to estimate the annual iron losses in a permanent magnet wind turbine generator, at the design stage. This type of result is vital for a machine design strategy in which minimization of the cost of energy is a design objective, but it has been shown to be potentially time-consuming. To that end, alternatives to direct simulation of all points of interest along the operating curve have been demonstrated.

Extrapolating a single simulation result is an acceptable practice in some cases, but is very dependent on the wind climate being considered. The results of this paper do suggest that if only one point is going to be simulated, it is a better idea to consider one where the machine is loaded, so that the effect of the armature current on the flux density throughout the iron is included.

Fitting the iron loss results from two simulations, in this case at no-load and full-load, gave robust agreement with usage of all simulated results to within a couple percent regardless of the wind regime.

For cases where it is more appropriate to focus on speed, but the accuracy of an FEA-based solution is still of interest, usage of a space-time transformation should be considered. This is especially true in the case where the design can be allowed to focus on a specific wind regime, since in this case extrapolating the results from one operational point does not result in a significant error.

\section{ACKNOWLEDGMENT}

This work is a part of the PhD project Wind Turbine Generators with Reduced Reliance on Rare Earth Metals, and the authors would like to thank DONG Energy for their gracious support of the project.

\section{REFERENCES}

[1] T. E. W. E. Association, "Wind in power, 2012 european statistics," The European Wind Energy Association, Tech. Rep., 2012.

[2] Siemens wind turbines. Siemens AG. Retrieved 18/03/2013 [Online]. Available: http://www.energy.siemens.com/hq/en/renewableenergy/wind-power/wind-turbines/

[3] Vestas turbine overview. Vestas Wind Systems A/S. Retrieved 18/03/2013. [Online]. Available: http://www.vestas.com/en/wind-powerplants/procurement/turbine-overview.aspx\#/vestas-univers
[4] Enercon wind turbines. Enercon GMBH. Retrieved 18/03/2013. [Online]. Available: http://www.enercon.de/en-en/Windenergieanlagen.htm

[5] Suzlon technology. Suzlon. Retrieved 18/03/2013. [Online]. Available: http://www.suzlon.com/products/13.aspx?11=2\&12=10\&13=20

[6] Alstom wind power. Retrieved 18/03/2013. [Online]. Available: http://www.alstom.com/power/renewables/wind/

[7] A. Grauers, "Efficiency of three wind energy generator systems," Energy Conversion, IEEE Transactions on, vol. 11, no. 3, pp. 650-657, 1996.

[8] A. Grauers, "Design of direct-driven permanent-magnet generators for wind turbines," Ph.D. dissertation, School of Electrical and Computer Engineering, Chalmers University of Technology, 1996.

[9] H. Polinder, F. F. Van der Pijl, G.-J. De Vilder, and P. J. Tavner, "Comparison of direct-drive and geared generator concepts for wind turbines," Energy conversion, IEEE transactions on, vol. 21, no. 3, pp. 725-733, 2006.

[10] H. Li, Z. Chen, and H. Polinder, "Optimization of multibrid permanentmagnet wind generator systems," Energy Conversion, IEEE Transactions on, vol. 24, no. 1, pp. 82-92, 2009.

[11] D. Kowal, L. Dupré, P. Sergeant, L. Vandenbossche, and M. De Wulf, "Influence of the electrical steel grade on the performance of the directdrive and single stage gearbox permanent-magnet machine for wind energy generation, based on an analytical model,' Magnetics, IEEE Transactions on, vol. 47, no. 12, pp. 4781-4790, 2011.

[12] H. Domeki, Y. Ishihara, C. Kaido, Y. Kawase, S. Kitamura, T. Shimomura, N. Takahashi, T. Yamada, and K. Yamazaki, "Investigation of benchmark model for estimating iron loss in rotating machine," Magnetics, IEEE Transactions on, vol. 40, no. 2, pp. 794-797, 2004.

[13] B. Wu, Y. Lang, N. Zargari, and S. Kouro, Power conversion and control of wind energy systems. Wiley-IEEE Press, 2011.

[14] I. Troen and E. L. Petersen, "European wind atlas," Roskilde: Riso National Laboratory, 1989, vol. 1, 1989.

[15] P. A. Hargreaves, B. C. Mecrow, and R. Hall, "Calculation of iron loss in electrical generators using finite element analysis," in Electric Machines \& Drives Conference (IEMDC), 2011 IEEE International. IEEE, 2011, pp. 1368-1373.

[16] Infolytica (2004), Team benchmark family: Problem 21 field loss in power transformer. Retrieved 26/03/2013. [Online]. Available: http://www-2004.infolytica.com/en/coolstuff/ex0134/

[17] Infolytica, "Infolytica livedocs," Infolytica Corporation, Tech. Rep., 2011.

[18] G. Y. Sizov, D. M. Ionel, and N. A. Demerdash, "Modeling and parametric design of permanent-magnet ac machines using computationally efficient finite-element analysis," Industrial Electronics, IEEE Transactions on, vol. 59, no. 6, pp. 2403-2413, 2012.

[19] D. M. Ionel and M. Popescu, "Ultrafast finite-element analysis of brushless pm machines based on space-time transformations," Industry Applications, IEEE Transactions on, vol. 47, no. 2, pp. 744-753, 2011.

[20] G. Y. Sizov, D. M. Ionel, and N. A. Demerdash, "A review of efficient fe modeling techniques with applications to pm ac machines," in Power and Energy Society General Meeting, 2011 IEEE. IEEE, 2011, pp. $1-6$.

[21] C. A. Hernandez-Aramburo, T. C. Green, and A. C. Smith, "Estimating rotational iron losses in an induction machine," Magnetics, IEEE Transactions on, vol. 39, no. 6, pp. 3527-3533, 2003.

[22] D. M. Ionel, M. Popescu, M. I. McGilp, T. Miller, S. J. Dellinger, and R. J. Heideman, "Computation of core losses in electrical machines using improved models for laminated steel," Industry Applications, IEEE Transactions on, vol. 43, no. 6, pp. 1554-1564, 2007. 